\title{
Single port training in Iraq-first uniportal video-assisted thoracoscopic surgery masterclass in Sulaimaniyah, Region of Kurdistan
}

\author{
Darya Akram Faqe Mahmood ${ }^{1,2}$, Diego Gonzalez-Rivas ${ }^{3,4,5}$ \\ ${ }^{1}$ Department of Thoracic and Vascular Surgery, Shorsh General Teaching Hospital, Sulaimaniyah, Iraq; ${ }^{2}$ Department of Cardiac Surgery, Slemani \\ Cardiac Hospital, Sulaimaniyah, Iraq; ${ }^{3}$ Department of Thoracic Surgery, Coruña University Hospital, Coruña, Spain; ${ }^{4}$ Minimally Invasive Thoracic \\ Surgery Unit (UCTMI), Coruña, Spain; ${ }^{5}$ Department of Thoracic Surgery, Shanghai Pulmonary Hospital, Shanghai, China \\ Correspondence to: Darya Akram Faqe Mahmood. Department of Thoracic and Vascular Surgery, Shorsh General Teaching Hospital, Sulaimaniyah, \\ Iraq. Email: darya_speed@yahoo.com.
}

\begin{abstract}
Video-assisted thoracoscopic surgery (VATS) has emerged more than 2 decades ago to treat thoracic diseases as a minimal invasive approach through multiple ports and utility incision. In the last decade, conventional VATS has been modified into uniportal (single port) VATS technique in which a $4 \mathrm{~cm}$ or smaller incision without a rib spreader is used to treat complex lung pathologies. Excellent outcome was achieved in terms of early recovery, reduced pain and shorter hospitalization period. The procedure was rapidly popularized worldwide due to its unique properties of being feasible, safe and effective. Like any novel technique in surgery, uniportal VATS demands a learning curve. Hands-on courses represent the best way to learn a new surgical technique, as it shortens learning curves and decreases complications. Herein, we present the first masterclass on uniportal VATS in Iraq which was conducted in December 2019 in the city of Sulaimaniyah/Region of Kurdistan in collaboration with Dr. Diego Gonzalez-Rivas, creator of uniportal VATS lobectomy. The course was attended by many thoracic surgeons and postgraduate students from different governorates of Iraq. Beside a lecture by Dr. Diego, surgery was performed on 2 cases; uniportal VATS lingulectomy for tuberculous bronchiectasis and biopsy of unresectable bronchogenic carcinoma.
\end{abstract}

Keywords: Video-assisted thoracoscopic surgery; lobectomy; uniportal; pulmonary tuberculosis; training

Received: 02 May 2020. Accepted: 02 June 2020; Published: 05 October 2020.

doi: $10.21037 /$ jovs-20-122

View this article at: http://dx.doi.org/10.21037/jovs-20-122

\section{Introduction}

Thoracic surgery has witnessed a dramatic change in the early 1990s when the open technique was challenged by minimal invasive technique as described by Giancarlo Roviaro. Dr. Roviaro did first lobectomy through multiple ports in the chest wall for lung cancer patient by videoassisted thoracoscopic surgery (VATS) (1). The technique showed clear advantages in terms of earlier recovery and less hospitalization and reduced pain (2).

Further refinement in surgical instruments, camera resolution and soft tissue cutting devices made the technique much more popular by shifting from three ports VATS to two ports and then to single port VATS. Initially the uniportal VATS was mostly used for minor procedures such as lung biopsy, wedge resection and treatment of pneumothorax. Thereafter, even complex pulmonary procedures were subsequently performed. Outcomes of the single port VATS were similar to multiple ports VATS but it had the added advantages of less pain and paresthesia, because it involved single small incision (2-4).

Uniportal VATS for lung biopsy has been done since 22 years ago (5). However, first VATS lobectomy through a single port was pioneered by Diego Gonzalez Rivas in 2010 (6). Thereafter owing to the potential advantage of uniportal VATS and its applicability for various lung procedures like bronchial sleeve lobectomy and pulmonary vascular reconstruction it became globally accepted. Currently uniportal VATS for major pulmonary resection can be done even in non-intubated patients by expert hands (7-9). 


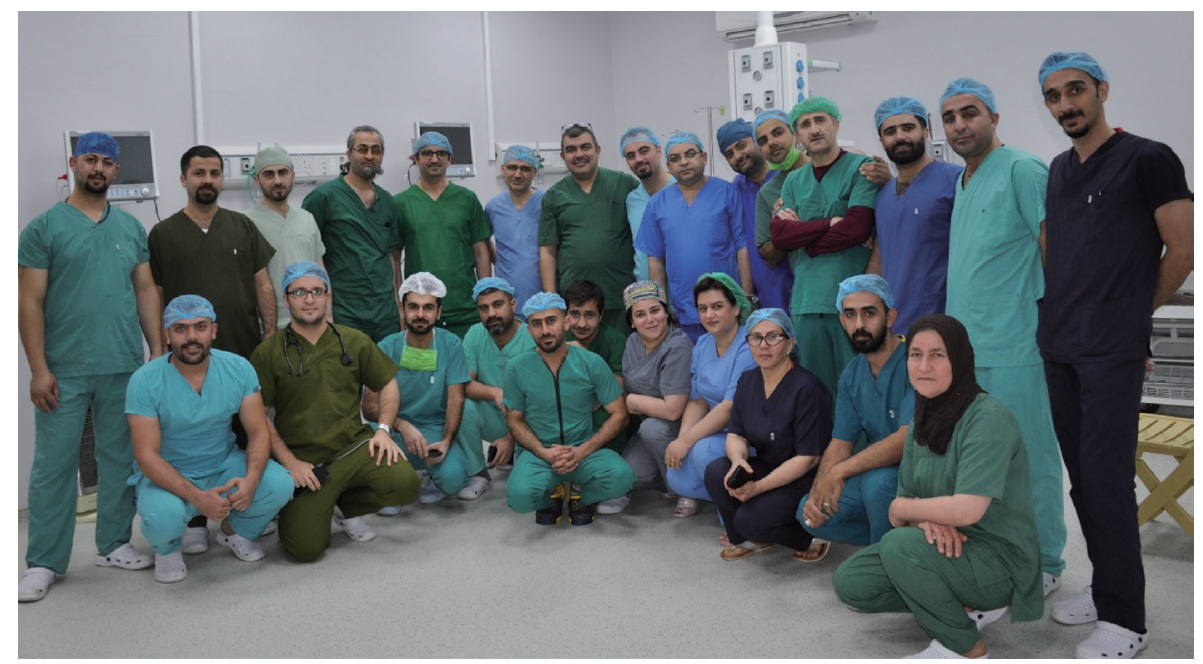

Figure 1 Participants of the uniportal VATS masterclass in Sulaimaniyah City, Iraq.

Herein, we present the first masterclass on uniportal VATS in Iraq which was conducted in December 2019 in the city of Sulaimaniyah/Region of Kurdistan in collaboration with Dr. Diego Gonzalez-Rivas, creator of uniportal VATS lobectomy. The 2-day course was attended by many thoracic surgeons and postgraduate students from different governorates of Iraq (Figure 1). Beside an interactive lecture on the evolution story of uniportal VATS by Dr. Diego (Figure 2), surgery was performed on 2 cases; uniportal VATS lingulectomy for tuberculous bronchiectasis (Figure 3, Video 1) and biopsy of unresectable bronchogenic carcinoma.

It is worthy to note that both lung cancer and pulmonary tuberculosis are common in our area. Bronchiectasis is defined as permanent dilatation of bronchi with destruction of the bronchial wall (10). Clinical picture varies greatly and may involve repeated respiratory infections alternating with asymptomatic periods or with chronic production of sputum (11). Diagnosis of bronchiectasis is based on clinical history and computed tomography (CT) scan findings. Early disease can be treated successfully by conservative methods while localized disease at any age should be surgically treated as surgery is associated with an acceptable morbidity and mortality (12). Uniportal VATS is a safe, feasible, and effective technique for selected bronchiectasis patients (13).

\section{Case presentation}

\section{Case 1}

A 41-year-old female patient, non-smoker, presented with 10 -years history of productive cough and mild hemoptysis unresponsive to conservative treatment. Workup revealed normal blood tests, pulmonary function tests besides sputum examination for Acid Fast Bacilli which was repeatedly negative. However, CT scan of the chest showed marked bronchiectasis of the lingua (Figure 4A) and fiberoptic bronchoscopy showed signs of chronic chest infection. The patient was scheduled for uniportal VATS lingulectomy after obtaining her informed consent. Anatomical lingulectomy was performed with a smooth postoperative course (Video 2). Histopathological exam of the resected specimen revealed pulmonary tuberculosis. Hence, anti-tuberculous drugs were started postoperatively. The patient was seen 4 months after surgery and she was doing very well.

\section{Case 2}

A 60-year-old non-smoker man presented himself with unresolved dry cough of 1-month duration. CT scan of the chest showed a big mass in the right lower lobe (RLL) extending to the middle lobe (ML) (Figure 4B). Bronchoscopy revealed a tumor bulging from RLL orifice extending to ML. Bronchoscopic biopsy was inconclusive. The patient was scheduled for exploration via uniportal VATS. PET scan was done but the result wasn't available at time of surgery. Unfortunately, surgical exploration revealed a big unresectable tumor mass invading the hilar vessels. A biopsy was obtained from right lower paratracheal lymph nodes. Frozen section exam revealed adenocarcinoma. 


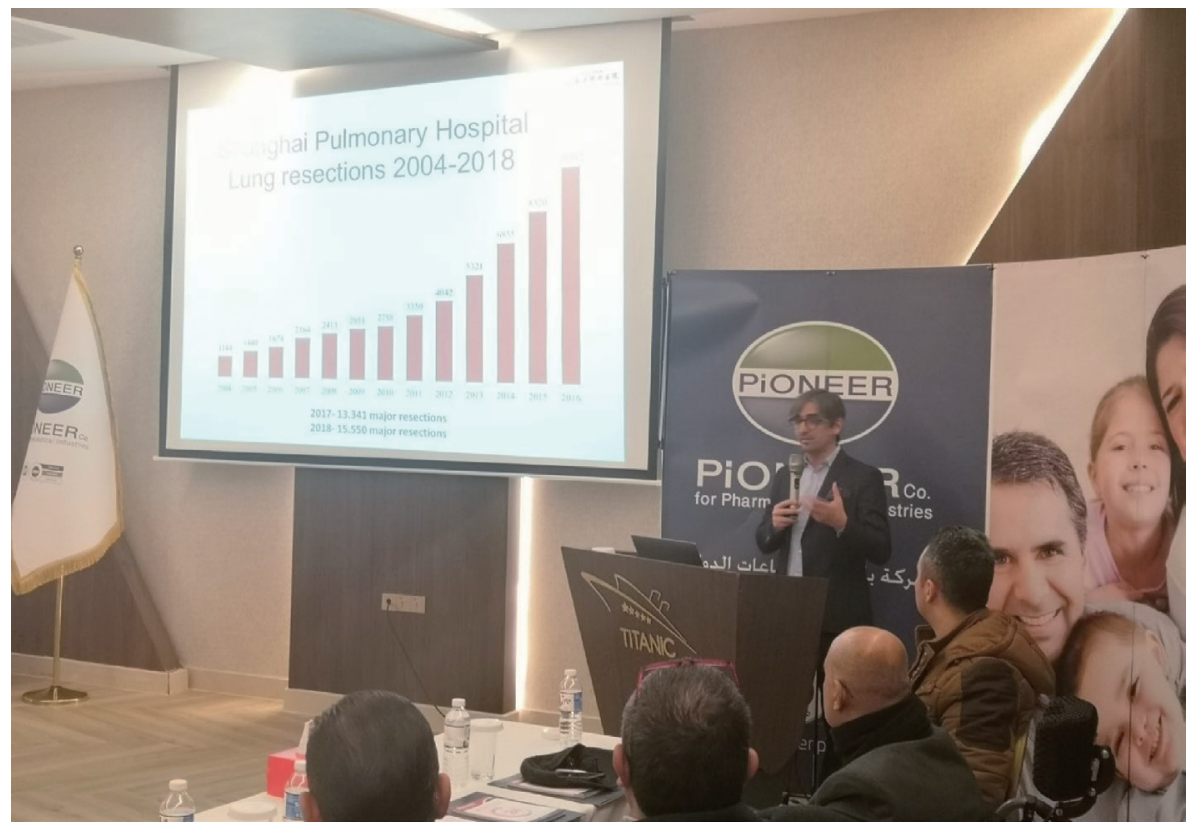

Figure 2 Dr. Diego lecturing about uniportal VATS in Sulaimaniyah, Iraq.

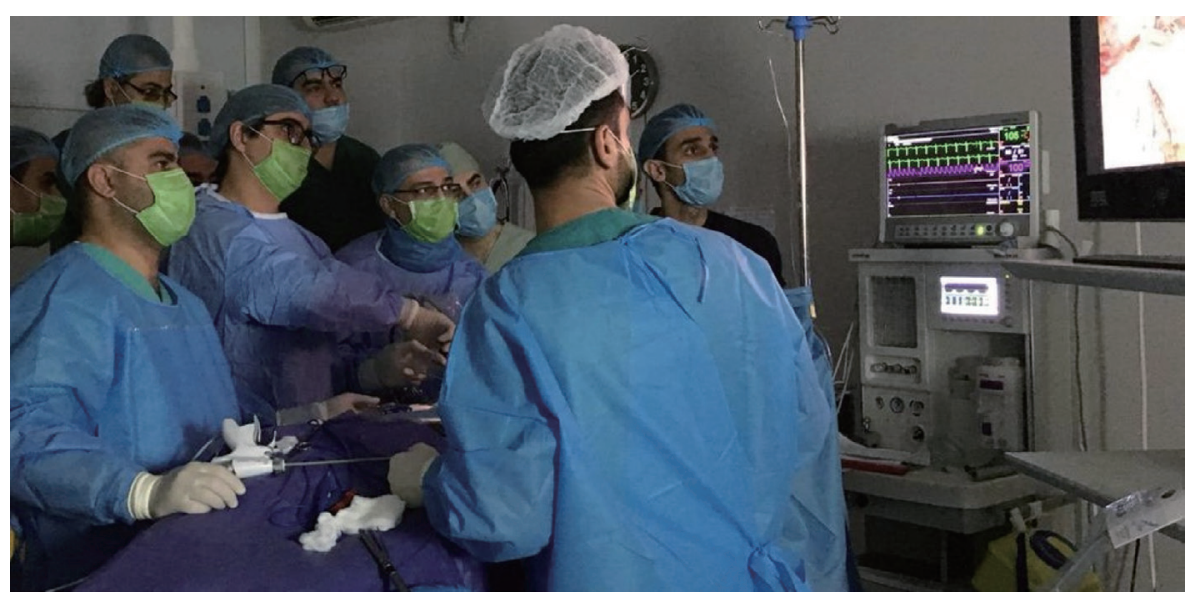

Figure 3 Dr. Diego Gonzalez-Rivas operating in Shorsh General Teaching Hospital.

Postoperatively, the patient was sent to multidisciplinary oncology team for further management.

\section{Equipment}

(I) A 10-mm 30-degree thoracoscopic camera system;

(II) Double articulating long shaft thoracoscopic instruments;

(III) Powered tissue staplers provided by;

(IV) Stapling loads;

(V) Vascular clipper and clips;
(VI) Energy source.

\section{Procedure}

Each Patient received general anesthesia via a double lumen endotracheal tube to enable deflation of the diseased lung. Electrocardiography leads were attached; central venous lines and urinary catheters were placed. Patients were then turned to the lateral decubitus position with a pillow between the legs and an axillary roll underneath the chest 


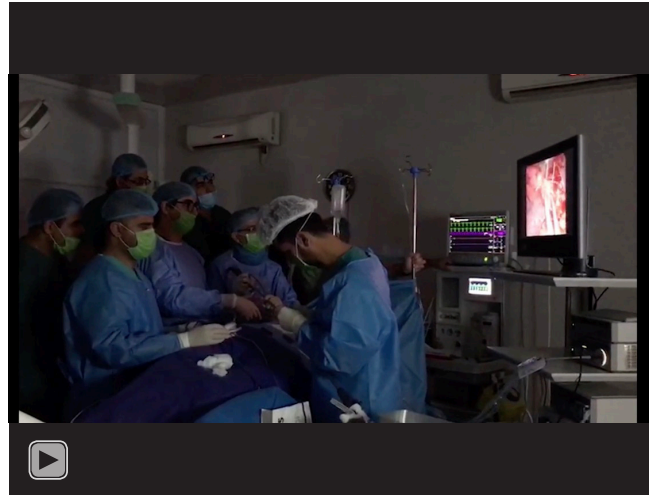

Video 1 Dr. Diego Gonzalez-Rivas and Dr. Darya Akram Faqe Mahmood, operating in Shorsh General Teaching Hospital, during the first uniportal VATS masterclass in Sulaimaniyah, Iraq.

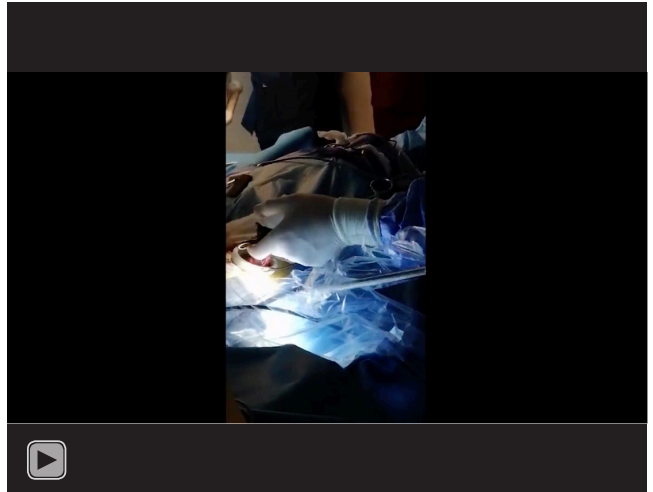

Video 2 Uniportal VATS lingulectomy for marked bronchiectasis of the lingula.
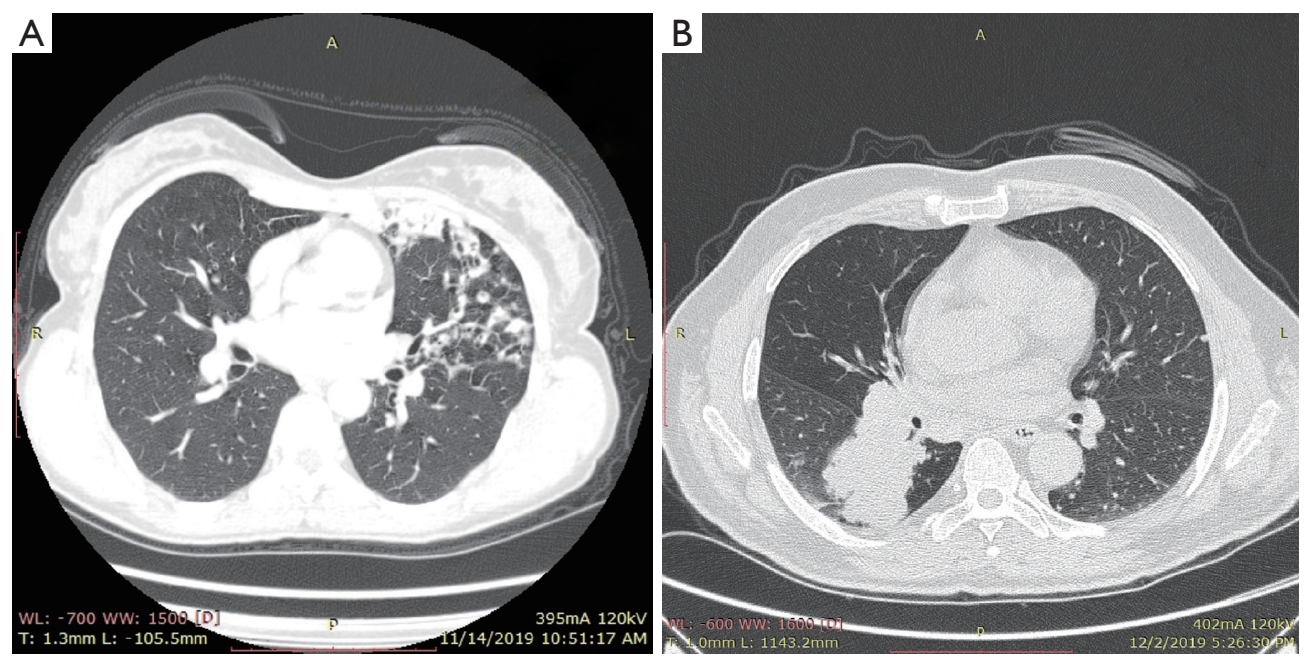

Figure 4 Uniportal VATS in Iraq, CT image of the cases. (A) CT scan of the chest showing bronchiectatic changes involving the lingular part of the left upper lobe. (B) CT scan of the chest showing big lower lobe mass.

at the level of the nipples to enlarge the intercostal space. A $3-4 \mathrm{~cm}$ incision was then made in the $5^{\text {th }}$ intercostal space close to the anterior axillary line. No rib spreader was used but a disposable wound retractor (protector) was placed in the wound for hemostasis and to keep the camera clean (Figure $5 A$ ). The resected specimen was delivered through the wound (Figure $5 B$ ). At the end of surgery, a single 24-Fr chest tube was introduced into the pleural cavity at the upper end of the incision (Figure 5C). An intercostal nerve block was done and the wound was closed in layers. Both patients were smoothly extubated and transferred to the ward. Chest tubes were removed in the second postoperative day and patients were discharged home on the third postoperative day.

\section{Discussion}

Uniportal VATS can be viewed as natural evolution towards minimizing invasiveness of multiport VATS. Uniportal VATS lobectomy showed favorable outcome compared to conventional multiport VATS for treatment of bronchiectasis (13) and lung cancer (14).

Shorsh General Teaching Hospital (SGTH) is a public hospital based in Sulaimaniyah, Iraq. In SGTH endoscopic surgery was begun in the late 1990s, mostly for biliary pathology and treating pneumothorax by clipping using 

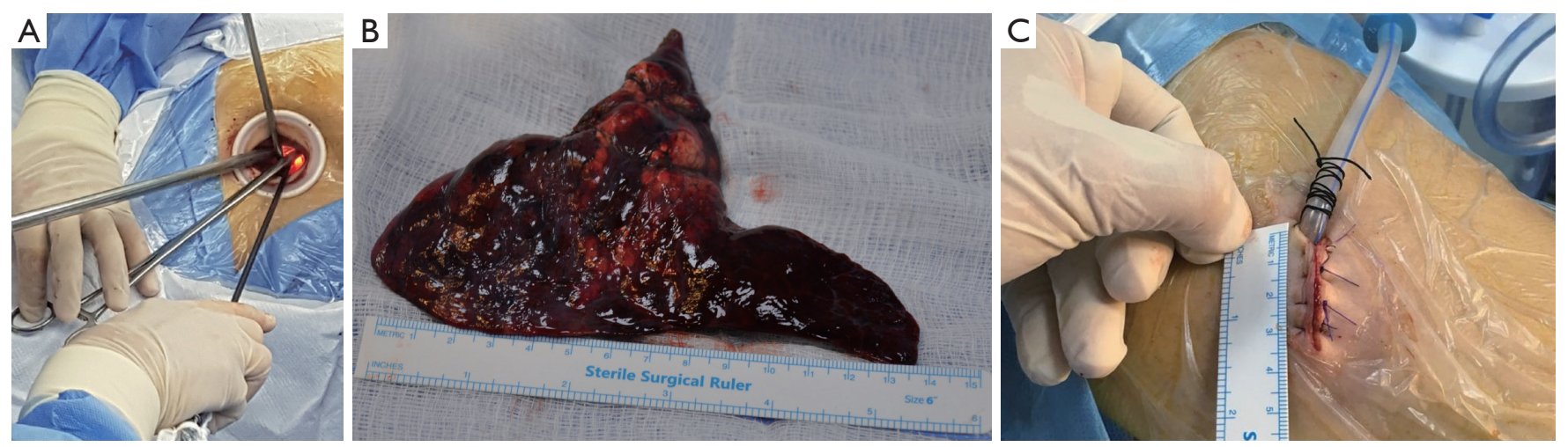

Figure 5 Uniportal VATS lingulectomy of the left upper lobe. (A) Disposable wound retractor and uniportal VATS instruments. (B) Resected lingular part of left upper lobe. (C) Size of the incision and position of the chest tube at upper end of the incision.

multiport technique. For many years our VATS practice didn't change. In January 2019, the first author of this paper had the opportunity to participate in the uniportal VATS training program directed by Dr. Diego Gonzalez-Rivas organized by Shanghai Pulmonary Hospital (SPH). SPH is a high-volume hospital in terms of number of patients operated upon (about 50 surgeries per day) and complexity of performed lung procedures.

After being familiar with the setup for uniportal VATS and having insight to its advantages, the author pursued the steps to learn uniportal VATS (learning from open approach to uniportal) (15). Subsequently, our department established the set up for uniportal VATS program and later on announced for uniportal VATS training program first masterclass in Kurdistan/Iraq.

The event took place in SGTH in Sulaimaniyah City in Kurdistan Region (North of Iraq) and the participants were thoracic surgeons from most cities of Iraq including the capital Baghdad. They were enlightened by the new technique and pleased to meet the creator of uniportal VATS lobectomy (Dr. Diego) who was eager to explain and teach the attendants how the technique can be reproduced. Furthermore, the participants joined an up-to-date lecture about uniportal VATS and its future application and robotic role in lung cancer surgery. Finally, the attendants expressed their excellent evaluation to the masterclass and utmost gratitude to Dr. Diego for sharing his experience, dedicating his time and efforts to teach novel science around the world.

\section{Guidance map to construct uniportal VATS program and to master the technique}

One of the most important prerequisites to start the program is to have the complete set of the equipment and surgical instruments aforementioned; each piece has its specific target action through this small incision.

Although we placed central venous catheters (CVCs) in our cases, neither CVCs nor peridural/epidural analgesia control systems are standard (16). Another important point is to have fully deflated lung during uniportal VATS by using a double lumen endotracheal tube. Should inappropriate lung deflation result from malpositioned tube, such a problem could be corrected by flexible bronchoscopy.

Learning from (multiport VATS to uniportal VATS) can be simplified by starting with thin female patients and lower lobectomies. For lower lobectomies, the posterior port should be removed and the camera placed in the posterior part of the utility incision along with bimanual instrumentation. For upper lobectomies place the camera through utility incision and use the inferior port first for stapling and instrumentation. Later, use the inferior port only to expose the lung and finally cancel the inferior port. Use vascular clips when there is no angle for stapler and always insert stapler with angulation for vascular division (15).

To learn from (open surgery to uniportal VATS), adopt the anterior small thoracotomy approach first. The thoracoscope is then added into the incision and observe the field through monitor. Gradually remove rib spreader and move the camera and instruments through $10-\mathrm{cm}$ incision. Lastly after gaining experience reduce the size of incision progressively (15). Visiting experienced uniportal VATS centers and attending wet-labs and hands-on courses can reduce the learning curve of this procedure (16).

The first consensus report from uniportal VATS interest group of the European society of thoracic surgeons defined and standardized the main procedural steps, the indication of 
VATS lobectomy, and elements to assist in future training (17). Hereby the latest recommendations are summarized:

(I) The incision should be done in the anterior or middle-anterior axillary line depending on the patient's built. However, the incision extension should be flexible and not be fixed to $4 \mathrm{~cm}$ but according to many other factors (18);

(II) The camera should be a 30-degree angle and $10-\mathrm{mm}$ diameter placed through the incision. The assistant should be on the same side of the surgeon;

(III) A single chest tube (size 24-Fr) is sufficient and should be placed through the same incision;

(IV) Tumor (T) sized $<5 \mathrm{~cm}$ (T1 and T2b) associated with lymph node (N) N0/N1 disease can be considered amenable to uniportal VATS lobectomy whereas chest wall involvement is not an absolute contraindication. Central location of a tumor invading hilar structures represents a relative contraindication as do previous ipsilateral thoracic surgery and pleurisy. Complete ipsilateral systemic lymph node dissection is the most appropriate associated procedure;

(V) For training in uniportal VATS lobectomy 50 cases are deemed the cut-off number required to overcome the learning curve. Moreover, thoracic surgeons should perform $>40$ cases annually to maintain uniportal operative skills.

\section{Conclusions}

Uniportal VATS lobectomy is one of the latest advancements in thoracic surgery. We were encouraged to adapt this rapidly spreading least invasive technique due to its efficacy, safety and feasibility. Learning curve can be reduced by attending uniportal VATS courses. Through such courses most of the tips and tricks related to the new technique would be unveiled by meeting the experts and pioneers in the field. Dr. Diego Gonzalez-Rivas is greatly thanked for serving the humanity and science via his countless courses to popularize this technique all over the world.

\section{Acknowledgments}

Funding: None.

\section{Footnote}

Provenance and Peer Review: The article was commissioned by the editorial office, Fournal of Visualized Surgery for the series "Teaching Uniportal VATS". This article has undergone external peer review.

Conflicts of Interest: Both authors have completed the ICMJE uniform disclosure form (available at https://jovs. amegroups.com/article/view/10.21037/jovs-20-122/coif). The series "Teaching Uniportal VATS" was commissioned by the editorial office without any funding or sponsorship. DGR served as the unpaid Guest Editor of the series and serves as an unpaid associate Editor-in-Chief of Fournal of Visualized Surgery. The authors have no other conflicts of interest to declare.

Ethical Statement: The authors are accountable for all aspects of the work in ensuring that questions related to the accuracy or integrity of any part of the work are appropriately investigated and resolved. All procedures performed in studies involving human participants were in accordance with the ethical standards of the institutional and/or national research committee(s) and with the Helsinki Declaration (as revised in 2013). Written informed consent was obtained from the patient for publication of this study and any accompanying images.

Open Access Statement: This is an Open Access article distributed in accordance with the Creative Commons Attribution-NonCommercial-NoDerivs 4.0 International License (CC BY-NC-ND 4.0), which permits the noncommercial replication and distribution of the article with the strict proviso that no changes or edits are made and the original work is properly cited (including links to both the formal publication through the relevant DOI and the license). See: https://creativecommons.org/licenses/by-nc-nd/4.0/.

\section{References}

1. Roviaro G, Rebuffat C, Varoli F, et al. Videoendoscopic pulmonary lobectomy for cancer. Surg Laparosc Endosc 1992;2:244-7.

2. Shah RD, D'Amico TA. Modern impact of video assisted thoracic surgery. J Thorac Dis 2014;6:S631-6.

3. Rocco G, Martin-Ucar A, Passera E. Uniportal VATS wedge pulmonary resections. Ann Thorac Surg 2004;77:726-8.

4. Jutley RS, Khalil MW, Rocco G. Uniportal vs standard three-port VATS technique for spontaneous pneumothorax: comparison of post-operative pain 
and residual paraesthesia. Eur J Cardiothorac Surg 2005;28:43-6.

5. Migliore M. Uniportal video-assisted thoracic surgery: twentieth anniversary. J Thorac Dis 2018;10:6442-5.

6. Gonzalez D, Paradela M, Garcia J, et al. Single-port videoassisted thoracoscopic lobectomy. Interact Cardiovasc Thorac Surg 2011;12:514-5.

7. Gonzalez-Rivas D, Fernandez R, Fieira E, et al. Uniportal video-assisted thoracoscopic bronchial sleeve lobectomy: first report. J Thorac Cardiovasc Surg 2013;145:1676-7.

8. Gonzalez-Rivas D, Delgado M, Fieira E, et al. Single-port video-assisted thoracoscopic lobectomy with pulmonary artery reconstruction. Interact Cardiovasc Thorac Surg 2013;17:889-91.

9. Gonzalez-Rivas D. Uniportal thoracoscopic surgery: from medical thoracoscopy to non-intubated uniportal videoassisted major pulmonary resections. Ann Cardiothorac Surg 2016;5:85-91.

10. Balkanli K, Genç O, Dakak M, et al. Surgical management of bronchiectasis: analysis and short-term results in 238 patients. Eur J Cardiothorac Surg 2003;24:699-702.

11. Vendrell M, de Gracia J, Olveira C, et al. Diagnosis and treatment of bronchiectasis. Spanish Society of Pneumology and Thoracic Surgery. Arch Bronconeumol 2008;44:629-40.

12. Al-Refaie RE, Amer S, El-Shabrawy M. Surgical treatment

doi: 10.21037/jovs-20-122

Cite this article as: Faqe Mahmood DA, Gonzalez-Rivas D. Single port training in Iraq-first uniportal video-assisted thoracoscopic surgery masterclass in Sulaimaniyah, Region of Kurdistan. J Vis Surg 2020;6:49. of bronchiectasis: a retrospective observational study of 138 patients. J Thorac Dis 2013;5:228-33.

13. Ocakcioglu I. Uniportal thoracoscopic treatment in bronchiectasis patients: preliminary experience. Wideochir Inne Tech Maloinwazyjne 2019;14:304-10.

14. Harris CG, James RS, Tian DH, et al. Systematic review and meta-analysis of uniportal versus multiportal videoassisted thoracoscopic lobectomy for lung cancer. Ann Cardiothorac Surg 2016;5:76-84.

15. Gonzalez-Rivas D, Fieira E, Delgado M, et al. Evolving from conventional video-assisted thoracoscopic lobectomy to uniportal: the story behind the evolution. J Thorac Dis 2014;6:S599-603.

16. Ismail $M$, Nachira D. Devising the guidelines: the concept of uniportal video-assisted thoracic surgeryinstrumentation and operatory room staff. J Thorac Dis 2019;11:S2079-85.

17. Bertolaccini L, Batirel H, Brunelli A, et al. Uniportal video-assisted thoracic surgery lobectomy: a consensus report from the Uniportal VATS Interest Group (UVIG) of the European Society of Thoracic Surgeons (ESTS). Eur J Cardiothorac Surg 2019;56:224-9.

18. Migliore M, Hirai K. Uniportal VATS: Comment on the consensus report from the uniportal VATS interest group (UVIG) of the European Society of Thoracic Surgeons. Eur J Cardiothorac Surg 2020;57:612. 